\title{
A study protocol for a randomized controlled trial on the
}

\section{prevention of atrial fibrillation after coronary artery bypass}

\section{grafting surgery using Tocotrienol, an isomer of Vitamin $\mathrm{E}$}

\section{derived from palm oil [version 1; peer review: 1 approved, 1}

\section{approved with reservations]}

\author{
Ahmad Farouk Musa (D1, Jeswant Dillon2, Mohamed Ezani Md Taib2, \\ Alwi Mohamed Yunus², Rusli Bin Nordin (D1, Yuen Kah Hay³ \\ ${ }^{1}$ Jeffrey Cheah School of Medicine and Health Sciences, Monash University Malaysia, Bandar Sunway, Selangor, Malaysia \\ ${ }^{2}$ Department of Cardiothoracic Surgery, National Heart Institute, Kuala Lumpur, Malaysia \\ ${ }^{3}$ School of Pharmaceutical Sciences, Universiti Sains Malaysia, Penang, Malaysia
}

\section{V1 First published: 22 Feb 2018, 7:215 \\ https://doi.org/10.12688/f1000research.12912.1 \\ Second version: 08 May 2018, 7:215 \\ https://doi.org/10.12688/f1000research.12912.2 \\ Latest published: 01 Aug 2018, 7:215 \\ https://doi.org/10.12688/f1000research.12912.3}

\section{Abstract}

Background: One of the most common complications following coronary artery bypass grafting (CABG) surgery is atrial fibrillation (AF), which contributes towards increasing morbidity and mortality, length of hospital stay (LoHS) and reduced quality of life (QoL) of patients.

Objectives: To determine whether the intake of Tocotrienol, a Vitamin $E$ isomer derived from palm oil, before and immediately following CABG prevents AF, reduces LoHS, and improves the QoL of patients. Protocol: The study is registered with the National Medical Research Register with a trial number NMRR-17-1994-34963 and designed as a prospective, randomized controlled trial (RCT) with parallel groups. The experimental group will receive two $200 \mathrm{mg}$ Tocotrienol capsules each day, while the control group will receive two identical placebo (palm Super Olein) capsules per day. ECG readings will be used to detect AF post operatively, LoHS will be measured by checking the records from the National Heart Institute Hospital register, and the health-related Quality of Life (HRQoL) analysis (the Malay version of the Short Form 36 Questionnaire) will be used to analyse QoL. The sample size was calculated to be 140 in each arm of the RCT for a power of 0.8 and a significance level of 0.05 .

Funding: HOVID Berhad funds this research project.

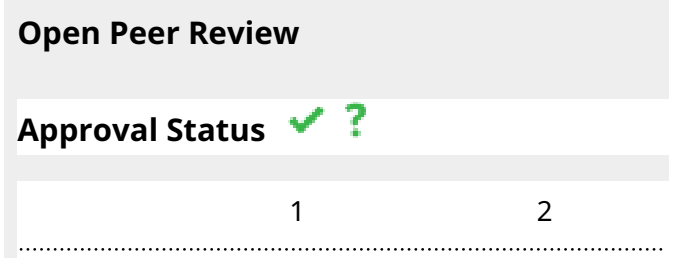

version 3

(revision)

01 Aug 2018

version 2

(revision)

08 May 2018

version 1

22 Feb 2018

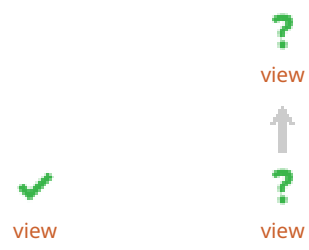

1. Whady Armino Hueb, University of São

Paulo, São Paulo, Brazil

Paulo Cury Rezende, University of São Paulo, Sao Paulo, Brazil

2. Olaf Stanger, University Hospital of Bern, Bern, Switzerland

Any reports and responses or comments on the 
Expected outcomes: The primary endpoint is the development of postoperative AF, whilst the secondary endpoints are the LoHS and HRQoL of patients post CABG.

Future implications: Prevention of AF and its complications such as cardiovascular or cerebrovascular events, especially stroke, is an important output. Malaysia is one of the biggest producers and exporters of palm oil and palm oil products. Thus, the possibility of marketing Tocotrienol, in reducing AF post CABG surgery, is a very important proposition indeed.

Trial number: NMRR-17-1994-34963

Keywords

Coronary Artery Bypass, Atrial Fibrillation article can be found at the end of the article.

Corresponding author: Ahmad Farouk Musa (farouk@monash.edu)

Author roles: Musa AF: Conceptualization, Data Curation, Formal Analysis, Funding Acquisition, Investigation, Methodology, Project Administration, Resources, Software, Supervision, Validation, Visualization, Writing - Original Draft Preparation, Writing - Review \& Editing; Dillon J: Project Administration, Resources, Supervision, Validation, Visualization; Md Taib ME: Project Administration, Resources, Validation, Visualization; Mohamed Yunus A: Project Administration, Resources, Validation, Visualization; Nordin RB: Project Administration, Supervision, Validation, Visualization, Writing - Original Draft Preparation, Writing - Review \& Editing; Kah Hay Y: Funding Acquisition, Project Administration, Resources, Supervision, Visualization

Competing interests: No competing interests were disclosed.

Grant information: HOVID Berhad (contact information: 121, Jalan Tunku Abdul Rahman, 30010 Ipon, Perak, Malaysia) sponsored this study (grant number, MMRD-MS-1801, awarded to the principle investigator, Ahmad Farouk Musa). The study design is solely developed by the main investigator. The sponsor, HOVID Berhad, supplies the materials for the research and the financial support.

The funders had no role in study design, data collection and analysis, decision to publish, or preparation of the manuscript.

Copyright: @ 2018 Musa AF et al. This is an open access article distributed under the terms of the Creative Commons Attribution License, which permits unrestricted use, distribution, and reproduction in any medium, provided the original work is properly cited.

How to cite this article: Musa AF, Dillon J, Md Taib ME et al. A study protocol for a randomized controlled trial on the prevention of atrial fibrillation after coronary artery bypass grafting surgery using Tocotrienol, an isomer of Vitamin E derived from palm oil [version 1; peer review: 1 approved, 1 approved with reservations] F1000Research 2018, 7:215

https://doi.org/10.12688/f1000research.12912.1

First published: 22 Feb 2018, 7:215 https://doi.org/10.12688/f1000research.12912.1 


\section{Introduction}

Atrial fibrillation (AF) after coronary artery bypass grafting $(\mathrm{CABG})$ surgery is common in clinical practice, with an incidence of up to $70 \%$. It has the potential to double the risk of mortality and is associated with a 6-fold increase in the risk of stroke ${ }^{1}$.

In a recent local study by Musa et al. on post-CABG patients, it was demonstrated that patients who developed postoperative AF had a prolonged Intensive Care Unit (ICU) stay and High Dependency Unit (HDU) stay, and a prolonged total hospital stay. This leads to an increase in resource utilization. With an increased rate of morbidity and mortality, preoperative prophylactic strategies are necessary to reduce the incidence of AF post-operatively and improve the Quality of Life (QoL) of patients $^{2}$

The financial burden in managing $\mathrm{AF}$ is huge, and many new innovative ideas and materials have been tested in order to reduce the occurrence of post-operative AF. One of the very promising but not widely studied treatment alternatives is the use of Tocotrienol, an isomer of vitamin E.

$\mathrm{AF}$ is thought to be initiated by rapid electrical activity arising from the muscular sleeves of pulmonary veins ${ }^{1,3}$. Multiple re-entrant wavelets, shorter refractory periods, conduction delays and fragmented electrograms maintain the arrhythmia ${ }^{1}$. If this arrhythmia continues, electrical remodeling occurs, which will further facilitate $\mathrm{AF}$ maintenance. The pathogenesis of post-operative AF is believed to be multifactorial. Previously it was thought to be caused by inflammatory pathways, but now it is known that oxidative stress plays a significant role ${ }^{4}$. Reactive oxygen species (ROS) are the cause of oxidative stress, and generate numerous pathological processes such as DNA damage, apoptosis, and cellular hypertrophy, as well as signal pathway modulation ${ }^{5}$.

Cardiac surgery is characterized by ischaemia and reperfusion injury, which leads to the release of ROS. This causes oxidative stress and initiates a systemic inflammatory response ${ }^{6}$. The extracorporeal circulation during on-pump surgery also activates inflammatory cells, increasing the oxidative stress ${ }^{7,8}$. Therefore it was always thought that an off-pump surgery would reduce the incidence of $\mathrm{AF}$.

Oxidative stress causes the depletion of endogenous antioxidants, and this results in oxidative damage. This oxidative stress is mainly the result of on-pump surgery that requires aortic cross-clamping and the bypass machine? .

Almost all cardiac centers have guidelines on the pharmacological management of postoperative $\mathrm{AF}^{10}$. But the challenge has always been how to prevent $\mathrm{AF}$ from happening. Efforts have been made to develop alternative preventive strategies. With the recent understanding of the pathogenesis of $\mathrm{AF}$ via the oxidative pathway, perhaps antioxidant vitamins would be a promising treatment.
Previous data have shown that both Vitamin E and $\mathrm{C}$ are known to have antioxidant properties, and have the ability to counter the action of free radicals ${ }^{11}$. Vitamin $\mathrm{E}$ is able to maintain membrane stability. It could also prevent the process of lipid peroxidation. Vitamin $\mathrm{C}$ removes water-soluble free radicals and acts synergistically with Vitamin E.

There have been numerous mechanistic studies on the role of antioxidant vitamins in the prevention of postoperative AF. However, the therapeutic potential is uncertain and there is no universally accepted protocol. It is interesting to note that Carnes et $a .^{12}$ reported a $16.3 \%$ incidence of postoperative $\mathrm{AF}$ in Vitamin C-treated patients compared to $34.9 \%$ in controls in their prospective study. In their meta-analysis, Harling et al. ${ }^{13}$ demonstrated a reduction in the incidence of postoperative AF and other forms of arrhythmia following antioxidant vitamin therapy, which remained significant when only randomized controlled trials (RCTs) were analyzed. They also showed that this reduction in incidence was not related to the bypass or crossclamp time, where there was no significant difference between the two-groups. Lastly, the results of the meta-analysis comparing the groups of anti-oxidants and control also demonstrated a reduced stay at the ICU and at the hospital. While this finding cannot be attributed solely to a reduction in postoperative AF, it is possible that this reduction in both ICU and hospital stay was a result of the reduced incidence of $\mathrm{AF}$ in the treatment group.

Rasoli et al. ${ }^{14}$ came to a similar conclusion that the use of antioxidant vitamins plays a role in the reduction of postoperative AF. However, they argued that the effect was somewhat variable, because both vitamins, $\mathrm{C}$ and $\mathrm{E}$, were weak anti-oxidants. They were limited in their capacity to cross the cell membrane in order to counter the superoxides.

We are proposing a more potent isomer of vitamin E, namely Tocotrienol, which is derived from palm oil, instead of the usual isomer of vitamin E, namely tocopherol, which was being used in all previous research. Tocotrienol has been proven to possess more potent antioxidant activity than tocopherols ${ }^{15}$. Laboratory studies have proven that Tocotrienol has the ability to scavenge peroxyl radicals in liposomes ${ }^{16}$. Tocotrienol is seen to be more evenly distributed in the phospholipid bilayer of the plasma membrane. It is also shown to have more efficient collision with radicals. These are some of the reasons why Tocotrienol is thought to have a more potent antioxidant properties ${ }^{15}$.

All these recent discoveries suggest the promising antioxidant properties for Tocotrienol. We hypothesize that it would be able to reduce the incidence of postoperative $\mathrm{AF}$ and subsequently reduce the complications associated with postoperative AF, along with the economic burden of prolonged intensive care stay and prolonged hospital stay. Overall, we also hypothesized that Tocotrienol will increase the health related quality of life (HRQoL) of patients. The potential value of marketing a national product that can be produced locally from palm oil and its potential uses, not only for patients, who are undergoing $\mathrm{CABG}$, but also for patients with standalone $\mathrm{AF}$, makes this an even more attractive product. 


\section{Protocol}

\section{Ethical statement}

The National Heart Institute Ethics Committee has approved the study protocol (IJNREC/20112017). The ethics committee will also serve as the data safety committee. Similar approval has been obtained from MUHREC - Monash University Human Research Ethics Committee - (9277) and from MREC - the Malaysian Research Ethics Committee. The study is registered with the National Medical Research Registry (NMRR), Ministry of Health, Malaysia, (NMRR-17-1994-34963) and will be carried out in accordance with the ethical principles outlined in the Malaysian Good Clinical Practice Guidelines.

Written informed consent for participation in the trial will be obtained from each participant on recruitment (Supplementary File 1).

The clinical data of patients enrolled in this study will be deidentified. No personal data will be disclosed to anyone outside of the National Heart Institute Ethics Committee.

This is Protocol Version 2.0 dated on 15 June 2017.

Trial registration: NMRR-17-1994-34963

Trial registry: National Medical Research Register, Malaysia

Trial registration date: $13^{\text {th }}$ March 2017

Study status: The study has not yet started at the time of submission of this manuscript since we are still waiting for the Clinical Trial Exemption (CTX) from the National Pharmaceutical Regulatory Agency (NPRA).

\section{Hypothesis}

Tocotrienol is a commercial product produced by Hovid Berhad, and is marketed as Tocovid Suprabio, containing $61.52 \mathrm{mg}$ alphaTocotrienol, $112.80 \mathrm{mg}$ gamma-Tocotrienol, $25.68 \mathrm{mg}$ deltaTocotrienol and 91.60IU alpha-tocopherol. Its availability in Malaysia has allowed this innovative and promising study to be conducted. We hypothesize that:

- Intake of Tocotrienol capsules before and immediately following $\mathrm{CABG}$ prevents $\mathrm{AF}$ post $\mathrm{CABG}$;

- Intake of Tocotrienol capsules before and immediately following $\mathrm{CABG}$ improves the QoL of patients post $\mathrm{CABG}$;

- Intake of Tocotrienol capsules before and immediately following CABG shortens the length of hospital stay (LoHS) of patients post $\mathrm{CABG}$.

\section{Objectives}

- To determine whether the intake of Tocotrienol capsules before and immediately following $\mathrm{CABG}$ prevents $\mathrm{AF}$ post $\mathrm{CABG}$.

- To determine whether the intake of Tocotrienol capsules before and immediately following CABG improves the QoL of patients post $\mathrm{CABG}$.

- To determine whether the intake of Tocotrienol capsules before and immediately following CABG shortens the LoHS of patients post $\mathrm{CABG}$.
Study design

Study site: National Heart Institute, Kuala Lumpur, Malaysia

The study is designed as a prospective, randomized controlled trial, with two parallel groups of the same demographics and comorbidities. The main aim is to look at the effect of Tocotrienol in reducing $\mathrm{AF}$ post $\mathrm{CABG}$.

Patients who are scheduled for CABG under the co-researchers at the National Heart Institute will be approached for their consent to be enrolled in this study once they are admitted to the wards. We will assign the patients enrolled in the study to one of two groups:

1. The control group: Routine $\mathrm{CABG}$ surgery procedure plus placebo containing palm Super Olein capsules (supplier: HOVID Berhad, Malaysia). The placebo is an identical-looking capsule that contains tocotrienolsstripped palm oil (termed palm Super Olein), which is also the non-active excipient in the treatment capsule. The choice of control is to mimic the treatment capsule as much as possible, without the active component of tocotrienols.

2. The study group: Routine CABG surgery procedure plus Tocotrienol capsules (supplier: HOVID Berhad, Malaysia)

For patients in the study group, Tocotrienol will be administered as two capsules of $200 \mathrm{mg}$ per day in two divided doses, and administration will start immediately after randomization and will continue until the first follow-up, which is normally six weeks after discharge.

Since there is no available data for preferred dose of treatment, we decided on two divided doses of Tocotrienol of one capsule twice daily of $200 \mathrm{mg}$ Tocovid. This dosage was estimated based on the regime used by Olaf Stanger et al. ${ }^{17}$ at the Department of Cardiac Surgery, Paracelcus Medical University Salzburg, Austria, where three ampoules of 45IE Vitamin E were used, with $30 \mathrm{mg}$ per ampoule or $90 \mathrm{mg}$ in total. Because in our study we would be using an oral preparation instead of an IV preparation as in the Austrian study, and absorption of Tocotrienol has been shown to be low and incomplete via the oral route, we estimated a higher dosage ${ }^{18}$. Considering the bioavailability of Tocotrienol can be as low 10-30\% if administered orally ${ }^{18}$; and taking into consideration that this is a pivotal study, it is reasonable to use the highest dose possible that is safe without any adverse effects. Many clinical studies with Tocotrienol have used $400 \mathrm{mg}$ daily in two divided doses and have been proven to be safe $^{19,20}$.

Tocovid will be administered right after randomization, and will continue after the patient is discharged until the first followup, which is normally about six-weeks later. If the patient is on prolonged ventilation in the ICU, Tocotrienol will be administered through a nasogastric tube. The intensive care nurses and cardiothoracic ward nurses will monitor compliance.

All patients undergoing CABG surgery, either or with valve surgery, will be included. Similarly, this study will include 
participants undergoing both on-pump surgery using cold potassium cardioplegia, and off-pump beating heart surgery.

All patients will be admitted to the intensive care unit (ICU) after CABG surgery, with close monitoring on one-patientone-nurse basis. They will then be transferred to a monitored high dependency unit (HDU) if their condition is stable, while some might be transferred straight back to the ward. Continuous rhythm monitoring will be performed using the 12-lead ECG for all patients in ICU and HDU. The monitoring will be continued for the first four to five postoperative days on the normal cardiothoracic wards using Holter monitors until the patients are discharged.

We will review the electrocardiographic (ECG) data on a daily basis. We will also review all the printouts of all abnormal rhythms, which will then be included in the clinical records. Additionally, an ECG will also be recorded when patients are symptomatic or when there is a suspicion of arrhythmia clinically. We will treat all AF episodes, in both the study arm and control arm, according to the protocols of the Cardiothoracic Department. The patients will be managed by the cardiothoracic surgeon and their team. The first-line drug used for treatment of new onset AF post-operatively by the Cardiothoracic team in the National Heart Institute (IJN) Hospital is Amiodarone, unless contraindicated, as a $300 \mathrm{mg}$ infusion over one hour to be followed by $900 \mathrm{mg}$ over the next 23 hours.

Patients of both the study and control arms will come back for follow-up at the Cardiothoracic Clinic at the IJN usually six weeks after discharge. They will also be advised to report to the Outpatient Department at the IJN if they develop any symptoms. During follow-up, all patients will be assessed routinely with blood work and a 12-lead ECG.

For the study flowchart, see Figure 1.

Hovid Berhad follow strict GLP Guidelines in the manufacturing process of Tocotrienols and matching placebo capsules. Each $200 \mathrm{mg}$ capsule of Tocotrienol will contain $61.52 \mathrm{mg}$ alphaTocotrienol, $112.80 \mathrm{mg}$ gamma-Tocotrienol, $25.68 \mathrm{mg}$ deltaTocotrienol and 91.60IU alpha-tocopherol. The placebo capsules will contain palm Super Olein oil.

\section{Randomization}

The study is designed as a randomized controlled trial (RCT), where patients with coronary artery disease requiring CABG surgery at the IJN will be prospectively and randomly divided into two parallel groups by means of computer generated numbers using Excel 2016 (Microsof, Redmont, WA, USA) in a block of 10 experimental to 10 matching controls at enrollment. The experimental group will receive Tocovid capsules whilst the control group will receive identical placebo capsules containing palm Super Olein oil.

The unblinded pharmacist will generate the allocation sequence to assign the participants to the interventions. Surgeon investigators will enroll the participants. The participants will then be assign randomly to either the study arm or the control arm based on the sequence assigned to them.

\section{Inclusion/exclusion criteria}

Inclusion criteria:

1. Males or females

2. More than 18 years of age

3. Elective, on-pump or off-pump CABG surgery of coronary artery revascularization, single or double procedure

Exclusion criteria:

1. Less than 18 years of age

2. Refusal to have surgery

3. Urgent or emergency surgery

4. Inability to give informed consent

5. Documented allergy to palm oil

Patients will be recruited from those who are scheduled electively for surgery under the Consultant Surgeons, JD, MEMT, and AMY, who are co-researchers in this project. All public and private patients are eligible to be enrolled in this study. Consent will be taken upon admission to the wards.

Patients can choose to withdraw from the study at any time. Subjects may be withdrawn if the investigator deems that it is detrimental or risky for the subject to continue. The patients who withdraw will not be replaced.

\section{Study endpoints}

As the primary study endpoint, we will look at the development of AF post-surgery. This will be documented with ECG. We take a 30 second duration as a cut-off point and define AF as when there is a loss of $\mathrm{p}$-waves and irregular ventricular rate or a confirmed atrial flutter ${ }^{21}$.

The secondary endpoint would include the LoHS after surgery, which will be obtained from the IJN registry, and the HRQoL. Three measurements will be used to determine the LoHS:

(1) Total number of days that the patients stay in the Intensive care unit (ICU)

(2) Total number of days in the high-dependency unit (HDU); and

(3) Total number of days in the hospital, overall ${ }^{21}$.

We will measure the HRQoL of patients using the validated Malay and English versions of Short-Form 36 Questionnaires $(\mathrm{SF}-36)^{22-24}$.

Since the researchers are blinded to the trial, we will be evaluating all endpoints, primary and secondary, independently. We will also review the patients' clinical records and all ECG tracings. In fact, all the trial participants, care-givers, outcome assessors and data analysts will be blinded to the study treatment allocation. An assigned pharmacist maintaining the randomisation list 


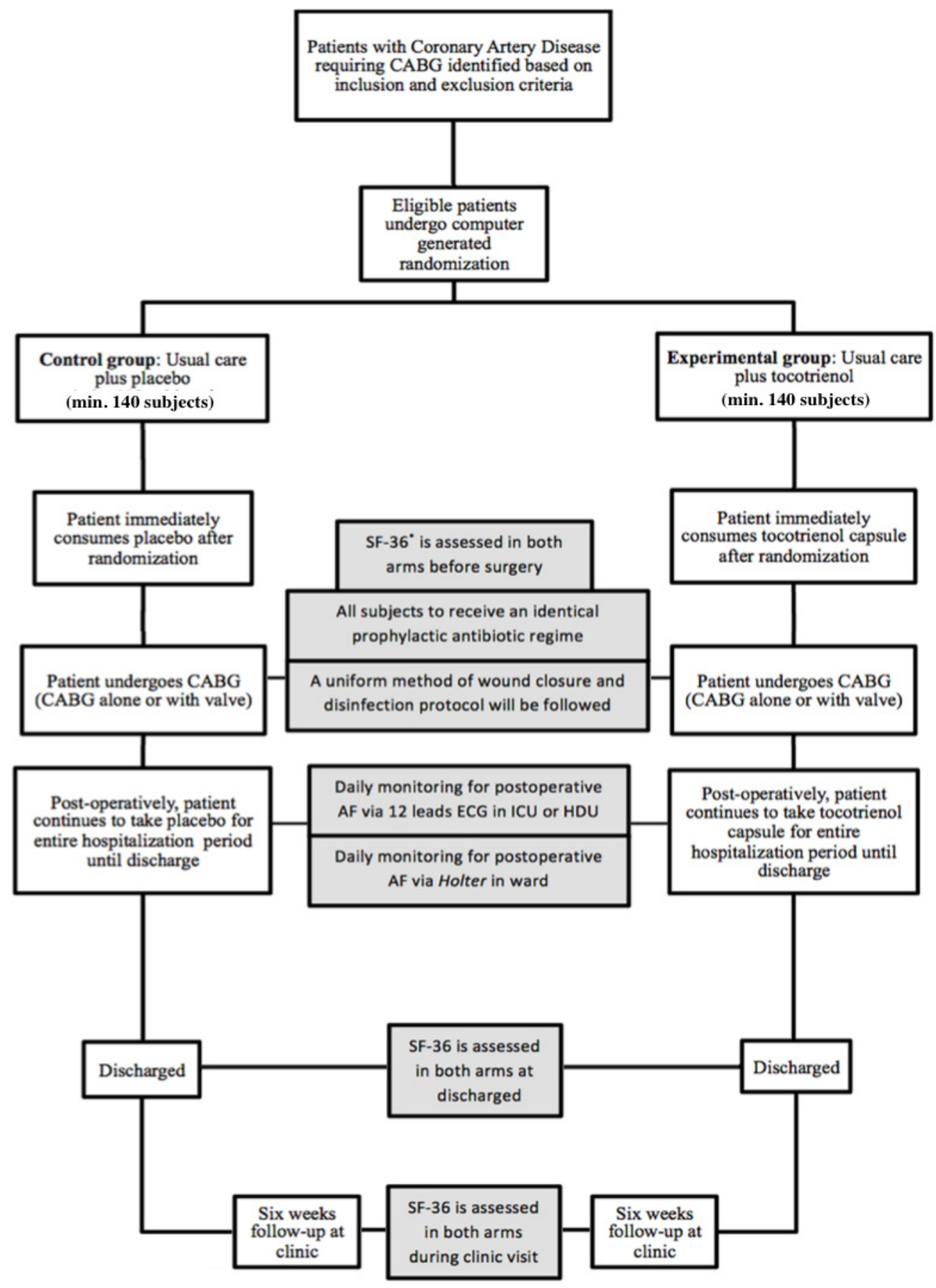

*SF-36 - Short Form 36 Questionnaire

Figure 1. Study flowchart. 
will be unblinded to the study treatment allocation. In the event of serious adverse events or emergency clinical treatment requiring knowledge of the study treatment, a request for unblinding shall be submitted to the pharmacist with reasonable justification for unblinding. The unblinding details and justification will be documented in the case record form. If unblinded, the participant will be withdrawn from the study in the event of unblinding, and post-treatment follow-up conducted as per protocol or as applicable. Where possible, the outcome assessor will remain blinded to all treatment allocation until end of study, even after participants are withdrawn from the study.

\section{Study procedures}

\section{Preoperative}

The two randomized groups, based in the IJN, will be matched according to sex, age, NYHA criteria, ejection fraction, and diabetic status. Operative and peri-operative conditions will also be similar for both groups. All subjects will receive an identical prophylactic antibiotic regime consisting of Cefazolin $2 \mathrm{gm}$ at induction and $1 \mathrm{gm} 12$ hourly for 48 hours. Gentamicin $2 \mathrm{mg} / \mathrm{kg}$ will also be given at induction.

\section{Intra-operative}

\section{- On-pump CABG}

A standard procedure of performing CABG will be done under general anaesthesia. Veins will be harvested from the legs and the left internal mammary will be taken down once the chest is opened. Titanium clips will be used to secure all side branches of saphenous veins and also the branches of the internal mammary artery. The patient will then be cannulated and placed on a heart-lung machine. Once the ascending aorta is cross-clamped, cold cardioplegic solution will be perfused in an ante-grade manner through the aortic root into the heart and the pericardial sac will be buried with ice sludge to create myocardial hypothermia.

The diseased coronary arteries will then be identified and arteriotomies will be performed beyond the level of blockages. The open ends of the saphenous veins and the internal mammary artery are sewn to the openings artertomies using Prolene 7/0 sutures. Once the distal anastomoses are constructed, proximal anastomoses will commence.

Once all the anastomoses are completed, the cross-clamp will be taken off and the heart-lung machine will then be gradually weaned off. Subsequently, the patient will be decannulated. Drainage catheters will be placed around the heart and temporary pacing will be sewn to the surface of the heart. Sternum will then be closed with steel wire and subcutaneous tissue and skin in the usual manner.

\section{- Off-pump CABG}

In this case, the bypass surgery will be done without the use of heart-lung machine. Surgery will be done on a beating heart. The procedure is similar to the on-pump surgery. Chest is opened and the left internal mammary artery is taken down. A stabilizer is placed on the heart to limit the motion of the heart. The anastomosis is done by sewing the open end of the internal mammary to the coronary artery, namely the left anterior descending artery. Chest is closed in the usual manner after placement of the drains and the temporary pacing wire.

\section{Post-operative}

Both groups will continue taking either two capsules of Tocotrienol or two capsules of placebo (palm Super Olein) daily for the entire hospitalization period until follow-up at six weeks after discharge. The capsules will be taken on a $b d$ - twicedaily dosing. If the patient is still ventilated in the cardiac ICU, the capsules will be broken and the content administered via a Ryle's tube.

After surgery, patients will be admitted to the ICU with close monitoring on one-patient-one-nurse basis and will be subsequently transferred to a monitored high dependency unit if their condition is stable. Continuous heart rhythm monitoring will be carried out using the 12-lead ECG. The monitoring will be continued for the first four to five postoperative days on the normal cardiothoracic wards using Holter monitors until the patients are discharged.

The electrocardiographic data will be stored for $24 \mathrm{~h}$ and reviewed on a daily basis by the cardiothoracic team involved in the research. The printouts of all abnormal rhythms will also be reviewed for any episodes of arrhythmia. All printouts will be included in the clinical records. Additionally, an ECG will also be recorded in case of symptoms or when arrhythmia is suspected on clinical grounds; AF episodes will always be treated under the direction of the attending cardiothoracic surgeon.

After discharge, all patients will be asked to report to the outpatient department of our institution in case of any relevant symptom. Additionally, all patients will be followed up six weeks after discharge; this will include physical examinaton and a 12-lead ECG measurement.

\section{Measurements}

\section{ECG readings to detect $A F$ post operatively}

Diagnostic criteria:

\section{Absent $P$ waves}

2. Evidence of fibrillation $(f)$ waves instead of $\mathrm{P}$ waves. These are irregular undulations of the base line in ECG.

3. Irregular R-R intervals

\section{Length of hospital stay (LoHS)}

This information will be obtained from the IJN registry of patients. Three measurements will be used to determine the LoHS:

(1) Total Cardiac ICU length of stay;

(2) Total HDU length of stay; and

(3) Total hospital length of stay.

\section{Health related quality of life (HRQoL)}

The analysis of HRQoL will be performed using the validated Short-Form 36 Questionnaire (SF-36). SF-36 has 
demonstrated its efficiency in clinical studies and has been proven to be generally acceptable to patients, consistent and a valid measure of health outcome. We will be using the validated Malay version of SF-36, which is a thirty-six items questionnaire, which measures QoL across eight domains that encompass both physical and mental.

The questionnaire will be distributed to both groups of patients before $\mathrm{CABG}$ and at discharge and six weeks during clinic visit postoperatively. The questionnaires are going to be administered using the questionnaire-interview approach.

\section{Sample size calculations}

We will use the PS Power and Sample Size Calculation Software version 3.1.2 for sample size calculation. We plan to study a continuous response variable from independent control and experimental subjects with 1 control per experimental subject.

Calculation of sample size in the present study (randomized controlled trial: RCT to rule out selection bias) requires precise specification of:

- The primary hypothesis of the study (Tocotrienol consumption reduces the incidence of post-operative atrial fibrillation in subjects that had undergone $\mathrm{CABG}$ )

- The method of analysis (using Relative Risk: RR).

We will also take into account the possibility of 'loss to follow-up' or attrition bias of subjects by analysing all subjects from the start to the completion of the study according to the groups that they were originally randomized. This is called an Intention-To-Treat (ITT) analysis ${ }^{25}$. To calculate the desired sample size based on the above consideration, we use the PS Power and Sample Size Calculation Software for sample size calculation ${ }^{26,27}$.

In the present RCT, estimated sample size for the primary endpoint (incidence of POAF) was computed on the basis of findings from Calo et al. ${ }^{4}$ In that study, the incidence of post-operative AF was $15.2 \%$ in the treatment group. Whereas in the placebo group, the incidence was $33.3 \%(\mathrm{RR}=15.2 / 33.3=0.46)$.

To account for possible subject withdrawal/non-compliance (attrition bias), we will adopt the ITT analysis so that subjects who are 'lost to follow-up'/non-compliant will be analyzed according to the groups that they were randomized at the beginning of the study. The formula suggested by Wittes ${ }^{25}$ is " $1 / 1-c$ " where $\mathrm{c}$ is the proportion that is lost to follow-up/did not comply. When $c=12 \%$, for a total enrollment of 260 patients. However, when $\mathrm{c}=20 \%$, the estimated sample size is increased to 278 (rounded to 280 ).

Using the PS Power and Sample Size Calculator ${ }^{26,27}$ with $\alpha$ equivalent to 0.05 and power $(1-\beta)$ is 0.8 , the estimated sample size based on the two proportions above is 130 intervention subjects and 130 control subjects (Fisher's exact test) if we assume the "lost to follow up" is $12 \%$. However, if we assume that the 'lost to follow up' is as high as $20 \%$, then the two proportions will be 140 (Intervention Group) and 140 (Control Group) with a total number of 280 patients.

\section{Statistical analysis}

IBM SPSS Statistics version 25.0 will be used to analyze the results. The ITT analysis will be used to analyze all outcome. The RR of the two-binomial proportion analysis will be used to test the occurrence of postoperative $\mathrm{AF}$ in the two treatment groups. The Kaplan-Meier method will be used to look at the cumulative risk. Log-rank test will compare the survival curves of the two treatment groups. Continuous variables will use mean $( \pm$ SD) while categorical variables will use frequencies (percentages). We will use unpaired Student $t$ test for continuous variables to look for differences between groups. And group differences will be examined by the chi-square or Fisher exact tests as appropriate for categorical variables. In case of an expected frequency of less than 5 in any cell in a $2 \times 2$ table, a Fischer Exact test will be applied. A $p$ value of less than 0.05 will be considered as statistically significant.

In order to find the predictors of AF after surgery, a stepwise multiple logistic regression analysis will be done. To examine the mean $( \pm \mathrm{SD})$ LoHS (number of days) differences between the two groups, we will use an unpaired Student-t test.

To examine the mean (+SD) QoL score differences between the two groups (pre-operative, six weeks, and three months), the one-way mixed-mode repeated measure ANOVA with posthoc multiple comparison test (between and within subject) of the two groups will be performed.

In order to look for the factors influencing the change in the quality of life after $\mathrm{CABG}$, we will perform the univariate simple logistic regression (SLogReg) initially, and examining the statistical significance of each independent variable such as the number of revascularization and duration of surgery on the outcome. Then, we will perform a multiple logistic regression (MLogReg) including variables with a level of significance less or equal to 0.25 in the univariate logistic regression and controlling for the effects of possible confounding variables (sex, age, NYHA Criteria, ejection fraction, and diabetic status). A p value of less than 0.05 will be taken as significant.

\section{Monitoring}

The Principal Investigator, AFM, will be responsible for ensuring participants' safety on a daily basis and for reporting Serious Adverse Events and Unanticipated Problems to his IJN Review Board (IJNRB) as required. The study statistician prepares reports that list adverse events, serious adverse events, deaths, and disease-or treatment-specific events required for monitoring body review in order to ensure good clinical care and identify any emerging trends. The IJNRB will act in an advisory capacity to the IJN Ethics Committee to monitor participants' safety, evaluate the progress of the study, to review procedures for maintaining the confidentiality of data, the quality of data collection, management, and analyses. 


\section{Data dessemination}

Data dissemination will be done via three ways. Firstly, via presentation at local and international conferences either in the form of posters and/or oral presentation. Secondly via writing for publication in scientific journals. Thirdly, via participation at international exhibitions and conventions on research and innovation.

\section{Study termination}

The sponsor has the full discretion to decide on the termination of the study at any time. Patients will be informed if the study is terminated and follow-up visits will be arranged if needed.

\section{Data Availability}

No data are associated with this article.

\section{Competing interests}

No competing interests were disclosed.

\section{Grant information}

HOVID Berhad (contact information: 121, Jalan Tunku Abdul Rahman, 30010 Ipon, Perak, Malaysia) sponsored this study (grant number, MMRD-MS-1801, awarded to the principle investigator, Ahmad Farouk Musa).

The study design is solely developed by the main investigator. The sponsor, HOVID Berhad, supplies the materials for the research and the financial support.

The funders had no role in study design, data collection and analysis, decision to publish, or preparation of the manuscript.

\section{Supplementary material}

Supplementary File 1: Informed consent form and participant information sheet.

Click here to access the data.

1. Nattel S, Allessie M, Haissaguerre M: Spotlight on atrial fibrillation-the 'complete arrhythmia'. Cardiovasc Res. 2002; 54(2): 197-203. PubMed Abstract | Publisher Full Text

2. Musa AF, Dillon J, Omar R: Atrial Fibrillation after Coronary Artery Bypass Grafting: A Restrospective Review of a Single Centre Experience. Heart Surg Forum. 2008; 2: 23

3. Markides V, Schilling RJ: Atrial fibrillation: classification, pathophysiology, mechanisms and drug treatment. Heart. 2003; 89(8): 939-943. PubMed Abstract | Publisher Full Text | Free Full Text

4. Banach M, Kourliouros A, Reinhart KM, et al:: Postoperative atrial fibrillation - what do we really know? Curr Vasc Pharmacol. 2010; 8(4): 553-572. PubMed Abstract | Publisher Full Text

5. Huang CX, Liu Y, Xia WF, et al:: Oxidative stress: a possible pathogenesis of atrial fibrillation. Med Hypotheses. 2009; 72(4): 466-467. PubMed Abstract | Publisher Full Text

6. $\mathrm{Kim} \mathrm{H}, \mathrm{Kim} \mathrm{KH}$ : Role of nitric oxide in oxidative damage in isolated rabbit gastric cells exposed to hypoxia-reoxygenation. Dig Dis Sci. 1998; 43(5): 1042-1049. PubMed Abstract | Publisher Full Text

7. Fontaine $\mathrm{D}$, Pradier $\mathrm{O}$, Hacquebard $\mathrm{M}$, et al:: Oxidative stress produced by circulating microparticles in on-pump but not in off-pump coronary surgery. Acta Cardiol. 2009; 64(6): 715-722. PubMed Abstract | Publisher Full Text

8. Matata BM, Sosnowski AW, Galiñanes M: Off-pump bypass graft operation significantly reduces oxidative stress and inflammation. Ann Thorac Surg. 2000; 69(3): 785-791.

PubMed Abstract | Publisher Full Text

9. De Vicchi E, Pala MG, Di Credico G, et al.: Relation between left ventricular function and oxidative stress in patients undergoing bypass surgery. Heart. 1998; 79(3): 242-247.

PubMed Abstract | Publisher Full Text | Free Full Text

10. Dunning J, Treasure T, Versteegh M, et al:: Guidelines on the prevention and management of de novo atrial fibrillation after cardiac and thoracic surgery. Eur J Cardiothorac Surg. 2006; 30(6): 852-872. PubMed Abstract | Publisher Full Text

11. Niki E: Interaction of ascorbate and alpha-tocopherol. Ann NY Acad Sci. 1987; 498: 186-199.

PubMed Abstract | Publisher Full Text

12. Carnes CA, Chung MK, Nakayama T, et al:: Ascorbate attenuates atrial pacinginduced peroxynitrite formation and electrical remodeling and decreases the incidence of postoperative atrial fibrillation. Circ Res. 2001; 89(6): E32-8. PubMed Abstract | Publisher Full Text

13. Harling L, Rasoli S, Vecht JA, et al.: Do antioxidant vitamins have an antiarrhythmic effect following cardiac surgery? A meta-analysis of randomised controlled trials. Heart. 2011; 97(20): 1636-1642. PubMed Abstract | Publisher Full Text

14. Rasoli S, Kakouros N, Harling L, et al.: Antioxidant vitamins in the prevention of atrial fibrillation: What is the evidence? Cardiol Res Prac. 2011; 2011: 164078. PubMed Abstract | Publisher Full Text | Free Full Text

15. Packer L, Weber S, Rimbach G: Molecular aspects of alpha-tocotrienol antioxidant action and cell signalling. J Nutr. 2001; 131(2): S369-S373. PubMed Abstract | Publisher Full Text

16. Serbinova $\mathrm{E}$, Kagan $\mathrm{V}, \mathrm{Had} \mathrm{D}$, et al: Free radical recycling and intramembrane mobility in the antioxidant properties of alpha-tocopherol and alpha-tocotrienol. Free Radic Biol Med. 1991; 10(5): 263-275. PubMed Abstract | Publisher Full Text

17. Stanger O, Aigner I, Schimetta W, et al: Antioxidant Supplementation Attenuates Oxidative Stress In Patients Undergoing Coronary Artery Bypass Graft Surgery. Tohoku J Exp Med. 2014; 232(2): 145-154. PubMed Abstract | Publisher Full Text

18. Yap SP, Yuen $\mathrm{KH}$, Lim AB: Influence of route of administration on the absorption and disposition of alpha-, gamma- and delta-tocotrienols in rats. $J$ Pharm Pharmacol. 2003; 55(1): 53-58. PubMed Abstract | Publisher Full Text

19. Gopalan $Y$, Shuaib IL, Magosso E, et al: Clinical investigation of the protective effects of palm vitamin $\mathrm{E}$ tocotrienols on brain white matter. Stroke. 2014; 45(5): 1422-1428 PubMed Abstract | Publisher Full Text

20. Magosso E, Ansari MA, Gopalan Y, et al:: Tocotrienols for normalisation of hepatic echogenic response in nonalcoholic fatty liver: a randomised placebocontrolled clinical trial. Nutr J. 2013; 12(1): 166-174. PubMed Abstract | Publisher Full Text | Free Full Text

21. Mozaffarian D, Marchioli R, Gardner T, et al: The $\omega-3$ Fatty Acids for Prevention of Post-Operative Atrial Fibrillation trial--rationale and design. Am Heart 2011; 162(1): 56-63.e3 PubMed Abstract | Publisher Full Text | Free Full Text

22. Jenkinson C: The SF-36 physical and mental health summary measures: an example of how to interpret scores. J Health Serv Res Policy. 1998; 3(2): 92-96. PubMed Abstract | Publisher Full Text 
23. Savelieva I, Camm AJ: Clinical relevance of silent atrial fibrillation: prevalence, prognosis, quality of life, and management. J Interv Card Electrophysiol. 2000; 4(2): 369-382.

PubMed Abstract | Publisher Full Text

24. Sararaks S, Azman AB, Low LL, et al.: Validity and reliability of the SF-36: the Malaysian context. Med J Malaysia. 2005; 60(2): 163-179.

PubMed Abstract

25. Wittes J: Sample size calculations for randomized controlled trials. Epidemiol
Rev. 2002; 24(1): 39-56.

PubMed Abstract | Publisher Full Text

26. Dupont WD, Plummer WD Jr: Power and sample size calculations: a review and computer program. Control Clinical Trials. 1990; 11(2): 116-28.

PubMed Abstract | Publisher Full Text

27. Dupont WD, Plummer WD Jr: Power and sample size calculations for studies involving linear regression. Control Clinical Trials. 1998; 19(6): 589-601.

PubMed Abstract | Publisher Full Text 


\section{Open Peer Review}

\section{Current Peer Review Status:}

\section{Version 1}

Reviewer Report 19 April 2018

https://doi.org/10.5256/f1000research.13999.r32234

(C) 2018 Stanger 0. This is an open access peer review report distributed under the terms of the Creative Commons Attribution License, which permits unrestricted use, distribution, and reproduction in any medium, provided the original work is properly cited.

\section{Olaf Stanger}

Clinic for Cardiovascular Surgery, University Hospital of Bern, Bern, Switzerland

The authors present the study protocol of a randomized controlled study to investigate the effect of a commercially available product (Tocovid Suprabio) that includes Tocotrienol, an Vitamin E isomer, against a placebo group, on the prevention of postoperative atrial fibrillation (AFib) in patients undergoing cardiac surgery.

The topic is of general interest. Postoperative AFib is common, is associated with complications and morbidity, thus consumes many resources and is, in part, potentially preventable. Because oxidative stress has been established as key mechanism, relatively cheap antioxidant treatment could be effective. The sample size under the current plan was calculated as 140 patients in each arm for a power of 0.8 .

The ambitious study plan has some important shortcomings that must be addressed.

1. As the authors correctly note, postoperative AFib is assumed to be a multifactorial event and may be triggered by mechanisms other than oxidative stress. That has severe impact on exclusion criteria and statistical power calculations, that is difficult to assess for me without further information.

2. The authors correctly note that the use of cardiopulmonary bypass is involved in ischemiareperfusion injury and radical oxygen species (ROS) generation. On page 3 it its stated that it is thought in consequence that off-pump surgery would reduce the incidence of AFib. I miss references, a clear statement whether or not this is thought to be a fact. In any case I would strongly suggest not to include off-pump patients and mix up the cohorts with onpump cases. It is difficult to precisely quantify the difference from a metabolic point, and it appears impossible to draw a conclusion on the effect of a given dose with unknown blood and tissue concentrations on different levels and sources of ROS.

3. What is known about the pharmacokinetics of the product? How quick and (in)complete must absorption be expected? What levels in blood and tissue can be achieved? Are interactions and competing drugs known? 
4. The authors "propose using a more potent isomer of vitamin E, namely Tocotrienol, instead of the usual Tocopherol" (page 3). However in describing the commercially available drug on page 4 (Tocovid Suprabio by Hocid Berhad) it is clearly expressed, that the capsule contains Tocopherol (!) besides Tocopherol. So how will the investigators ever know which component, if any, was effective?

5. The authors aim at assessing the health-related quality of life in patients undergoing cardiac surgery; by definition a very sick group of elderly patients with usually many comorbidities and with much drug intake. This aim is an extremely difficult matter involving many critical sources of definition, assessment tools (including SF-36), error and bias and I doubt that a meaningful result can be obtained.

6. According to the study protocol patients are required to take the capsules "before" and immediately following CABG. Before is defined as "immediately after randomization" on page 4. It is quite normal that even elective cardiac surgery can not always be planned precisely on the exact day and hour, must be delayed for medical or organizational reasons. Therefore there will be patients with various time intervals of capsule intake and supposedly different blood and tissue concentrations of the study medication!

Capsules intake should last for approx 6 weeks until the first follow-up. Nearly all cases of AFib occur within the first 4 days postoperative. It is extremely rare to observe first-time AFib later than that. Although the long intake is ambitious how do the investigators control full compliance?

7. Again, bioavailibility is an issue. The authors state on page 4 that bioavailibility of oral Tocotrienol is as low as 10-30\%; how long before surgery must the drug be given to guarantee an effective availability in all patients of the non-placebo group? Is the trial controlled for interference with other drugs, malabsorption, possibly even gastric resections or concomitant intestinal disease etc that may prevent the study drug to be taken up effectively?

8. Definitely exclude patients undergoing concomitant valve surgery (page 4)! Operating times are longer, the heart will be OPEN with further exposure to ROS-producing surfaces and valve pathologies, stretch and surgical maneuvers are all known to cause and influence postoperative AFib. Up to $7 \%$ of patients following aortic valve replacement require permanent pacemakers anyway! Again, I would also suggest not to mix on- and off-pump patients.

9. Holter ECG, very essential, will be monitored for the first 5 days, which makes sense. The study drug is given for approx 6 weeks. There will be no continuous ECG monitoring between discharge and follow-up. Unless the patient would be symptomatic and decides to see a physician who may be able to make a documentation, investigators are likely never to know whether there was an episode of AFib at any time between 1 and 6 weeks after surgery.

10. I miss any information on potential PREoperative AFib assessment; or at least the exclusion of patients with AFib or other arrhythmias before surgery! 
11. And authors should exclude patients with vitamin or antioxidant intake before randomization! Check for all medications as many are known to exert antioxidant side effects.

12. Finally I would consider it extremely valuable to obtain blood samples and analyse various ROS and vitamin concentrations (or at least the antioxidant capacity) to proof that any clinical outcome is directly linked to a mechanism as hypothesized. Otherwise, any outcome can be spurious and by chance, in principle and by definition.

Is the rationale for, and objectives of, the study clearly described?

Yes

Is the study design appropriate for the research question?

Partly

Are sufficient details of the methods provided to allow replication by others? Partly

Are the datasets clearly presented in a useable and accessible format?

Not applicable

Competing Interests: No competing interests were disclosed.

I confirm that I have read this submission and believe that I have an appropriate level of expertise to confirm that it is of an acceptable scientific standard, however I have significant reservations, as outlined above.

Author Response 02 May 2018

Ahmad Farouk Musa, Monash University Malaysia, Bandar Sunway, Malaysia

The authors present the study protocol of a randomized controlled study to investigate the effect of a commercially available product (Tocovid Suprabio) that includes Tocotrienol, an Vitamin $\mathrm{E}$ isomer, against a placebo group, on the prevention of postoperative atrial fibrillation (AFib) in patients undergoing cardiac surgery.

The topic is of general interest. Postoperative AFib is common, is associated with complications and morbidity, thus consumes many resources and is, in part, potentially preventable. Because oxidative stress has been established as key mechanism, relatively cheap antioxidant treatment could be effective. The sample size under the current plan was calculated as 140 patients in each arm for a power of 0.8 .

The ambitious study plan has some important shortcomings that must be addressed.

1. As the authors correctly note, postoperative AFib is assumed to be a multifactorial event and may be triggered by mechanisms other than oxidative stress. That has severe impact on exclusion criteria and statistical power calculations, that is difficult 
to assess for me without further information.

Thank you Prof for your comments and suggestions. In the revised version of this study, we will include a few other exclusion criteria namely poor LV, off-pump surgery, combined valve surgery and currently on or indicated for long-term corticosteroid treatment.

1. The authors correctly note that the use of cardiopulmonary bypass is involved in ischemia-reperfusion injury and radical oxygen species (ROS) generation. On page 3 it its stated that it is thought in consequence that off-pump surgery would reduce the incidence of AFib. I miss references, a clear statement whether or not this is thought to be a fact. In any case I would strongly suggest not to include off-pump patients and mix up the cohorts with on-pump cases. It is difficult to precisely quantify the difference from a metabolic point, and it appears impossible to draw a conclusion on the effect of a given dose with unknown blood and tissue concentrations on different levels and sources of ROS.

Our assertion that off-pump surgery would reduce the incidence of AF post-CABG was due to the fact that the extracorporeal circulation during on-pump surgery would activate the inflammatory cells thus increasing the oxidative stress that would later lead to AF. This was also acknowledged by Hashemzadeh $\mathrm{K}$ et al. in their paper published by the Journal of Cardiovascular and Thoracic Research 2013;5(2),45-49, with the title "Does off-pump coronary artery bypass reduce the prevalence of atrial

fibrillation?, though we did not cite it. However, in the revised version of this Study Protocol, we have omitted Off-pump surgery.

1. What is known about the pharmacokinetics of the product? How quick and (in)complete must absorption be expected? What levels in blood and tissue can be achieved? Are interactions and competing drugs known?

Gan et al. published in Scientific Reports 2017; 7(1): p11542 on "Effect of palm-based tocotrienols and tocopherol mixture supplementation on platelet aggregation in subjects with metabolic syndrome: a randomised controlled trial" showed that a two-week supplementation of the same product and same dosing schedule resulted in plasma levels of approximately $0.58 \mathrm{ug} / \mathrm{mL}$. And Patel et al. published in the Journal of Nutrition 2012; 142(3):513-519 on "Oral Tocotrienols are transported to human tissues and delay the progression of the model for end-stage liver disease score in patients" demonstrated that oral supplementation of tocotrienols in surgical patients up to four weeks before surgery significantly increased the tissue concentrations in blood, skin, adipose, brain, cardiac muscle, and liver. In our study, plasma tocotrienols levels will be measured to indicate the levels in the blood pre and post-surgery, and also on follow-up to determine if the blood levels are sufficient to exert clinical effects at the end of the study. And Tocotrienols have not been noted to have any interference with other drugs so far.

1. The authors "propose using a more potent isomer of vitamin E, namely Tocotrienol, instead of the usual Tocopherol" (page 3). However in describing the commercially available drug on page 4 (Tocovid Suprabio by Hocid Berhad) it is clearly expressed, that the capsule contains Tocopherol (!) besides Tocopherol. So how will the investigators ever know which component, if any, was effective?

The proposed investigation uses a commercially available product of natural mixed tocotrienols. Almost all natural sourced and marketed tocotrienols products contain a small fraction of tocopherols. In palm oil-derived tocotrienols, the composition been 
established as approximately $80 \%$ tocotrienols, $20 \%$ tocopherols. The investigation aims to evaluate the commercially available product (mixed tocotrienols, tocopherols and phytosterols) in the proposed effect on AF, and rather than isolated tocotrienols effect. We believe, the overall anti-oxidative effect may in part be contributed by the action of tocopherols with enhanced bioactivity from the much higher fraction of tocotrienols. The effects could potentially be synergistic to result in a clinically significant outcome.

1. The authors aim at assessing the health-related quality of life in patients undergoing cardiac surgery; by definition a very sick group of elderly patients with usually many co-morbidities and with much drug intake. This aim is an extremely difficult matter involving many critical sources of definition, assessment tools (including SF-36), error and bias and I doubt that a meaningful result can be obtained.

We acknowledged the concerns regarding the use of health-related quality of life (HRQOL) instruments, such as the SF-36, to document the QOL of principally sick, elderly patients undergoing CABG surgery. In order to improve the validity and reliability of the SF-36, a Malay version of the SF-36 has been developed and tested. Results suggest that the Malay-SF-36 is a valid and reliable tool to measure the HRQOL of patients undergoing CABG surgery at the National Heart Institute (IJN) Kuala Lumpur, Malaysia. Both English and Malay validated versions of SF-36 will be administered to the patients before surgery, at discharge, and at 6-weeks follow-up visit. A one-way repeated measure ANOVA analysis will be undertaken to assess changes in HRQOL across the three time points.

1. According to the study protocol patients are required to take the capsules "before" and immediately following CABG. Before is defined as "immediately after randomization" on page 4. It is quite normal that even elective cardiac surgery can not always be planned precisely on the exact day and hour, must be delayed for medical or organizational reasons. Therefore there will be patients with various time intervals of capsule intake and supposedly different blood and tissue concentrations of the study medication!Capsules intake should last for approx 6 weeks until the first follow-up. Nearly all cases of AFib occur within the first 4 days postoperative. It is extremely rare to observe first-time AFib later than that. Although the long intake is ambitious how do the investigators control full compliance?

The National Heart Institute has a protocol that must be adhered known as CABG Clinical Pathway where patients should not stay longer than seven days before surgery. Though we initially planned that patients should at least be on study medication three days before surgery, after randomisation, we find it rather difficult to achieve since there will be patients who will be admitted just two days prior to surgery at our centre. And to ensure that all study patients have a rather stable and sustainable level of the study medication, we have made it compulsory that the study patients to take the study drugs a minimum of seven days prior to surgery. This could be achieved by randomising the patients upon referral to the Cardiothoracic Wards for surgery once they have consented to be enrolled. We would take their blood samples at the beginning of randomization, pre-op, on Day-4 post-op, at discharge and on the first follow-up at six-weeks.

The variability in dosing duration will be noted and supported with pre-surgery blood levels of tocotrienols. We will control for the dosing duration during analysis, if 
necessary. However, due to the short half-life of tocotrienols, the small variation of dosing duration is not expected to have a significant impact in the blood levels of tocotrienols.

And we also realised the fact that almost all AF occurred during the first four days post-operatively and our previous retrospective study at our centre showed that the median time for the development of AF was 45 hours post-CABG where the majority developed within three days post-surgery with the second day being most common. But we would like patients to consume the study medication until the first follow-up which is six-weeks after discharge and will try to ensure compliance by asking the research assistants to remind the patients intermittently at home and that their blood will be taken upon their follow-up visit and pill-counting will be done as well. The patients will be informed before discharge about the blood taking and pill counting to ensure that they have good compliance.

Apart from first-time AF, we will continue to monitor the safety endpoints, including symptoms requiring medical treatment during the post-discharge supplementation of 6 weeks. This will allow us to evaluate the effects of continued supplementation in post-CABG recovery.

1. Again, bioavailibility is an issue. The authors state on page 4 that bioavailibility of oral Tocotrienol is as low as 10-30\%; how long before surgery must the drug be given to guarantee an effective availability in all patients of the non-placebo group? Is the trial controlled for interference with other drugs, malabsorption, possibly even gastric resections or concomitant intestinal disease etc that may prevent the study drug to be taken up effectively?

The investigational product is formulated with a patented delivery system that provides $100-200 \%$ enhanced bioavailability, of mixed tocotrienols. If taken on fasted state, tocotrienols, as with all oil-soluble vitamins, have been shown to have poor and erratic absorption due to dependence on food status. However, this is resolved using the enhanced absorption delivery system. The subjects are proposed to initiate supplementation at least one week from surgery in order to ensure levels of tocotrienols in the blood and tissues. All concomitant medication and medical history will be noted upon enrolment and throughout the study to evaluate any possible interaction effects on the study outcomes. The medical history and plasma levels will help us determine if gastrointestinal issues or any drugs may cause significant impact on the absorption and final clinical outcome.

1. Definitely exclude patients undergoing concomitant valve surgery (page 4)! Operating times are longer, the heart will be OPEN with further exposure to ROS-producing surfaces and valve pathologies, stretch and surgical maneuvers are all known to cause and influence postoperative AFib. Up to 7\% of patients following aortic valve replacement require permanent pacemakers anyway! Again, I would also suggest not to mix on- and off-pump patients.

Yes Prof, we will exclude the concomitant valve surgery patients and Off-pump CABG. This is reflected in the revised version of the Study Protocol.

1. Holter ECG, very essential, will be monitored for the first 5 days, which makes sense. The study drug is given for approx 6 weeks. There will be no continuous ECG monitoring between discharge and follow-up. Unless the patient would be 
symptomatic and decides to see a physician who may be able to make a documentation, investigators are likely never to know whether there was an episode of AFib at any time between 1 and 6 weeks after surgery.

Based on our previous retrospective study, only $15 \%$ of our patients developed AF after more than three days post-surgery. Yes, we will never know if they would develop AF at home unless they present themselves to our centre again. But considering all our patients were discharged in sinus rhythm, we do not anticipate that they would develop AF after being discharged from the hospital.

1. I miss any information on potential PREoperative AFib assessment; or at least the exclusion of patients with AFib or other arrhythmias before surgery!

Yes, all patients documented with AF pre-operatively will be excluded from the study.

1. And authors should exclude patients with vitamin or antioxidant intake before randomization! Check for all medications as many are known to exert antioxidant side effects.

All pre-operative medications will be documented and patients on any form of antioxidant vitamins pre-operatively will be excluded. We will include this in our revised Study Protocol.

1. Finally I would consider it extremely valuable to obtain blood samples and analyse various ROS and vitamin concentrations (or at least the antioxidant capacity) to proof that any clinical outcome is directly linked to a mechanism as hypothesized. Otherwise, any outcome can be spurious and by chance, in principle and by definition.

Apart from anti-oxidative mechanisms, we postulate that tocotrienols may potentially inhibit the HMGCo-reductase to attenuate AF, similar to statins. We will consider including relevant blood biomarkers to support the findings of the study. However, due to transient effect of oxidative stress and whether blood levels are representative of cardiac condition remains a concern.

Competing Interests: No competing interests were disclosed.

Reviewer Report 02 March 2018

https://doi.org/10.5256/f1000research.13999.r31123

(C) 2018 Hueb W et al. This is an open access peer review report distributed under the terms of the Creative Commons Attribution License, which permits unrestricted use, distribution, and reproduction in any medium, provided the original work is properly cited.

Whady Armino Hueb

Heart Institute (InCor), University of São Paulo, São Paulo, Brazil

Paulo Cury Rezende

Heart Institute (InCor) Medical School, University of São Paulo, Sao Paulo, Brazil 
These reviewers suggest changing the title to:

"Hypotheses, rationale, design, and methods for evaluation of a randomized controlled trial using Tocotrienol, an isomer of Vitamin E derived from palm oil, on the prevention of atrial fibrillation after coronary artery bypass grafting surgery".

We appreciate the opportunity to review the manuscript "A study protocol for a randomized controlled trial on the prevention of atrial fibrillation after coronary artery bypass grafting surgery using Tocotrienol, an isomer of Vitamin E derived from palm oil".

In this study, the authors aimed to test Tocotrienol, an isomer of Vitamin E derived from palm oil in the prevention of atrial fibrillation after myocardial revascularization surgery.

The study is well designed, well written and well detailed in definitions and methods.

However, some serious methodological failures should be avoided in order not to irreversibly compromise the study.

It is known that myocardial revascularization surgery with the use of extracorporeal circulation adds non-physiological effects with direct damage to the myocardium.

Such effects include: cardiac arrhythmias, low cardiac output, SIRS, thrombocytopenia, among others. The systemic inflammatory process is usually treated with corticosteroids. Thus, authors cannot ignore the differences between on-pump or off-pump.

As suggestions, these reviewers recommend the following care in patient selection and data analysis.

1. The surgery should be only on-pump or just off-pump.

2. Patients with valvular heart disease cannot be included in patient selection. Aortic stenosis is often accompanied by myocardial hypertrophy, and Mitral insufficiency accompanies an increase in the left atrium. Both conditions facilitate the development of atrial fibrillation.

3. The authors should include only patients with preserved left ventricular function. Ventricular dysfunction is one of the leading causes of AF.

4. The use of corticosteroids should be prespecified and, preferably, avoided because of its known anti-inflammatory actions.

5. Timing from randomization and consequently beginning of Tocotrienol or placebo to surgery could be a relevant issue. A short time since medication start and surgery could compromise a possible significant effect of the drug on AF development.

6. A Holter monitoring must be installed only in the first 72 hours. It is known that the most sensitive moment for the onset of AF is the immediate postoperative period. However, the use of the Holter monitoring for a longer period, even if uncomfortable, may be useful.

7. In statistical analysis, the stepwise multiple logistic regression analysis should include atrium data such as size and/or volume, and procedure-related variables, such as use of 
inotropes and other vasoactive drugs, cross-clamp and cardiopulmonary bypass time. All of these variables might be associated with the occurrence of AF after revascularization procedures and should be included in the final model. On the other hand, overfitting should be avoided.

8. In the multiple logistic regression model for the analysis of the factors influencing the change in quality of life after CABG, the authors should also include in the model angina data such as angina presence or frequency. In order to assess whether Tocotrienol is associated to better quality of life measures, it would be helpful if the authors control for angina relief after surgery.

Is the rationale for, and objectives of, the study clearly described?

Yes

Is the study design appropriate for the research question?

Yes

Are sufficient details of the methods provided to allow replication by others?

Yes

Are the datasets clearly presented in a useable and accessible format?

Yes

Competing Interests: No competing interests were disclosed.

We confirm that we have read this submission and believe that we have an appropriate level of expertise to confirm that it is of an acceptable scientific standard.

Author Response 30 Mar 2018

Ahmad Farouk Musa, Monash University Malaysia, Bandar Sunway, Malaysia

Thank you Prof Hueb and Prof Rezende. Your comments were highly beneficial for the improvement of this project. Since we have not started this project yet and waiting for CTX Clinical Trial Exemption from NPRA - National Pharmaceutical Regulatory Agency, we would include all the suggestions in the Study Protocol which will be resubmitted for Ethical Clearance. And to make reading easier, I'll copy the comments and reply immediately below.

1. The surgery should be only on-pump or just off-pump.

Since the National Heart Institute is an on-pump center, only a small minority of less than $\mathbf{5 \%}$ were off-pump cases. This was based on the previous retrospective research that we have done on atrial fibrillation after CABG. Since we have not started this study yet and waiting for the CTX -Clinical Trial Exemption approval, we will include this in the Exclusion Criteria. Refer: A retrospective study on atrial fibrillation after coronary artery bypass grafting surgery at the National Heart Institute, Kuala Lumpur. F1000Research 2018; 7:164. 
2. Patients with valvular heart disease cannot be included in patient selection. Aortic stenosis is often accompanied by myocardial hypertrophy, and Mitral insufficiency accompanies an increase in the left atrium. Both conditions facilitate the development of atrial fibrillation.

In our center, less than $10 \%$ of cases are combined surgery based on the same retrospective research cited above. And based on that the same paper we cited above, we noticed that there was a higher incidence of AF in patients with combined surgery although the association was not significant after adjustment. However since some paper noticed this correlation, we have no issue in classifying this under the Exclusion Criteria.

3. The authors should include only patients with preserved left ventricular function. Ventricular dysfunction is one of the leading causes of AF.

Similarly we also noticed a statistically significant difference in the previous study we cited above in between patients with poor left ventricular function and preserved left ventricular function. And since these patients only constitute less than $10 \%$ (for $E F<30 \%$ ) and less than $1 \%$ (for $E F<20 \%$ ) of our total patients load of about 1800 CABG patients per year, we will also exclude them from the study.

4. The use of corticosteroids should be prespecified and, preferably, avoided because of its known anti-inflammatory actions.

It is not customary for our surgeons especially the co-researchers to add corticosteroids in the pump. We will specify that all patients must not be given corticosteroids. Patients with a history of long-term corticosteroids treatment at screening will be excluded.

5. Timing from randomization and consequently beginning of Tocotrienol or placebo to surgery could be a relevant issue. A short time since medication start and surgery could compromise a possible significant effect of the drug on AF development.

This is an issue which we have thought and discussed for quite some time. We wanted to load the patient a minimum of two days prior to surgery or preferably three days. The Institute protocol of Clinical Pathway for CABG does not allow patients to stay longer than 72 hours prior to surgery. So we will ensure that all patients will receive their tocotrienol right after randomization and not less than two days prior to surgery.

6. A Holter monitoring must be installed only in the first 72 hours. It is known that the most sensitive moment for the onset of AF is the immediate postoperative period. However, the use of the Holter monitoring for a longer period, even if uncomfortable, may be useful.

We do not normally use Holter for our patients post-operatively but we will 
monitor them via continuous ECG monitoring. Our previous study cited above showed that $95 \%$ of patients developed AF within the first 48 hours. We will monitor them using continuous ECG monitoring for 72 hours post-operatively.

7. In statistical analysis, the stepwise multiple logistic regression analysis should include atrium data such as size and/or volume, and procedure-related variables, such as use of inotropes and other vasoactive drugs, cross-clamp and cardiopulmonary bypass time. All of these variables might be associated with the occurrence of AF after revascularization procedures and should be included in the final model. On the other hand, overfitting should be avoided.

We will include data on atrium size and/or volume, use of inotropes and other vasoactive drugs, cross-clamp and cardiopulmonary bypass time in the study proforma and the final model. We will modify and strengthen the proforma we used when collecting the data for our retrospective research before. Using multiple logistic regressions, we will choose the most parsimonious model and avoid overfitting of the model. Calibration of the model will be evaluated with the Hosmer-Lemeshow goodness-of-fit test, and discrimination with the Areas Under the ROC Curve (AUC) will be used as a guide towards model fitting strategy.

8. In the multiple logistic regression model for the analysis of the factors influencing the change in quality of life after CABG, the authors should also include in the model angina data such as angina presence or frequency. In order to assess whether Tocotrienol is associated to better quality of life measures, it would be helpful if the authors control for angina relief after surgery.

Yes, the frequency of angina will be included and controlled in the multiple logistic regression models for factors influencing the change in the quality of life after CABG.

Thank you, Professors. Your comments are highly appreciated.

Competing Interests: There is no competing interest. 
The benefits of publishing with F1000Research:

- Your article is published within days, with no editorial bias

- You can publish traditional articles, null/negative results, case reports, data notes and more

- The peer review process is transparent and collaborative

- Your article is indexed in PubMed after passing peer review

- Dedicated customer support at every stage

For pre-submission enquiries, contact research@f1000.com 